\title{
FUNCTIONS WHICH OPERATE ON THE REAL PART OF A FUNCTION ALGEBRA
}

\author{
OSAMU HATORI
}

\begin{abstract}
Recently S. J. Sidney [5] has shown that a "highly nonaffine" function $h$ on an interval cannot operate by composition on the real part of a nontrivial function algebra. In this paper, we obtain the general result by considering the case in which $h$ is not "highly nonaffine".
\end{abstract}

1. Introduction. Let $A$ be a function algebra on a compact Hausdorff space $X$ and $h$ a nonaffine function on an interval $I$. We say that $h$ operates by composition on $\operatorname{Re} A$ if $h \circ u \in \operatorname{Re} A$ whenever $u \in \operatorname{Re} A$ has range in $I$. We consider a conjecture: If $h$ operates by composition on $\operatorname{Re} A$, then we have $A=C(X)$. The theorem of $\mathrm{J}$. Wermer [7] is equivalent to the conjecture for $h(t)=t^{2}$. A. Bernard [1] proved the conjecture for $h(t)=|t|$. S. J. Sidney [5] obtained results for the cases that $h$ is "highly nonaffine" or continuously differentiable. Our purpose is to show the following theorem.

THEOREM. Suppose that $A$ is a function algebra on a compact Hausdorff space $X$ and that $h$ is a nonaffine continuous function on an interval I. If $h$ operates by composition on $\operatorname{Re} A$, then we have $A=C(X)$.

If $h$ is nonaffine on every nondegenerate subinterval of $I$, then $h$ is "highly nonaffine". So without loss of generality we may assume that $I=[-1,1], h=0$ on $[-1,0]$ and $h$ is not affine on any open subinterval of $I$ containing 0 .

$\operatorname{Re} A$ is a Banach space with the usual quotient norm

$$
N(u)=\inf \{\|f\|: f \in A, \operatorname{Re} f=u\} .
$$

We denote $f \mid F$ the restriction of a function $f \in C(X)$ to a subset $F \subset X$. For nonempty disjoint compact subsets $F_{1}$ and $F_{2}$ of $X$ we denote

$$
\begin{aligned}
& (\operatorname{Re} A)_{1}=\left\{u \in C_{R}\left(F_{1}\right): \exists \hat{u} \in \operatorname{Re} A, \hat{u} \mid F_{1}=u\right\}, \\
& (\operatorname{Re} A)_{1}^{2}=\left\{u \in C_{R}\left(F_{1}\right): \exists \hat{u} \in \operatorname{Re} A, \hat{u}\left|F_{1}=u, \hat{u}\right| F_{2}=0\right\} .
\end{aligned}
$$

For $u \in(\operatorname{Re} A)_{1}$, we define

$$
N_{1}(u)=\inf \left\{N(\hat{u}): \hat{u} \in \operatorname{Re} A, \hat{u} \mid F_{1}=u\right\} .
$$

For $u \in(\operatorname{Re} A)_{1}^{2}$, we define

$$
N_{1}^{2}(u)=\inf \left\{N(\hat{u}): \hat{u} \in \operatorname{Re} A, \hat{u}\left|F_{1}=u, \hat{u}\right| F_{2}=0\right\} .
$$

Received by the editors December 16, 1980.

1980 Mathematics Subject Classification. Primary 46J10.

Key words and phrases. Function algebra, real part, ultraseparate. 
Then $(\operatorname{Re} A)_{1}$ and $(\operatorname{Re} A)_{1}^{2}$ are complete with respect to the norms $N_{1}(\cdot)$ and $N_{1}^{2}(\cdot)$, respectively.

2. Lemmas. Let $x$ be a point of $X$. We say that the function $h$ operates weak-boundedly at $x$ if there exist a $\delta>0$, an $\varepsilon>0$, a compact neighborhood $F_{x}$ of $x$, and a compact subset $F_{0}$ of $X$, which is disjoint from $F_{x}$, with the following property: $h \circ u \in(\operatorname{Re} A)_{x}$ and $N_{x}(h \circ u)<\varepsilon$ for each $u \in(\operatorname{Re} A)_{x}^{0}$ with $N_{x}^{0}(u)<\delta$.

LEMMA 1. Suppose that $h$ operates by composition on $\operatorname{Re} A$. Then $h$ operates weak-boundedly at each point of $X$ except for at most finitely many points.

Proof. Suppose that the lemma fails. Then there exists a countable subset $\left\{x_{n}\right\}$ of $X$ with the following two properties: (1) Each $x_{n}$ has a compact neighborhood $F_{n}$ such that $\left(\mathrm{Cl}\left(\cup_{k \neq n} F_{k}\right)\right) \cap F_{n}=\varnothing$ for each positive integer $n$. (2) $h$ does not operate weak-boundedly at each point $x_{n}$. Let $F_{m(n)}$ denote $\mathrm{Cl}\left(\cup_{k \neq n} F_{k}\right)$ for each $n$. ( $m(n)$ is the index depending on $n$.) Since $h$ does not operate weak-boundedly at $x_{n}$, there exists a $u_{n} \in(\operatorname{Re} A)_{n}^{m(n)}$ such that $N_{n}^{m(n)}\left(u_{n}\right)<1 / 2^{n}$ and $N_{n}\left(h \circ u_{n}\right)>n$ for each $n$. There exists a $\hat{u}_{n} \in \operatorname{Re} A$ such that $\hat{u}_{n}\left|F_{n}=u_{n}, \hat{u}_{n}\right| F_{m(n)}=0$ and $N\left(\hat{u}_{n}\right)<$ $1 / 2^{n}$ for each $n$. So $\sum_{n=1}^{\infty} \hat{u}_{n}=\hat{u} \in \operatorname{Re} A$ and $h \circ \hat{u} \in \operatorname{Re} A$ and $\hat{u} \mid F_{n}=u_{n}$ for each $n$. Thus $N(h \circ \hat{u}) \geqslant N_{n}\left((h \circ \hat{u}) \mid F_{n}\right)=N_{n}\left(h \circ u_{n}\right)>n$ for each $n$. This contradicts $h \circ \hat{u} \in \operatorname{Re} A$.

Lemma 2. Let $F_{0}$ and $F_{1}$ be nonempty disjoint compact subsets of $X$. Then $(\operatorname{Re} A)_{0}^{1}$ is an ultraseparating Banach function space with respect to the norm $N_{0}^{1}(\cdot)$.

Proof. For each $p \in \beta\left(N \times F_{0}\right)$ the functional $u \mapsto \bar{u}(p)$ on $C_{R}\left(F_{0}\right)$ is linear and multiplicative, so there is a unique $x_{p} \in F_{0}$ such that $\bar{u}(p)=u\left(x_{p}\right)$ for all $u \in C_{R}(X) \cdot\left(\bar{u}=\left(u_{n}\right)\right.$ where $u_{n}=u$ for all $n$.) Let us take $p, q \in \beta\left(N \times F_{0}\right)$ and $p \neq q$. We shall find $g \in l^{\infty}(N, \operatorname{Re} A)$ such that $g(\tilde{x})=0$ for $\tilde{x} \in \beta\left(N \times F_{1}\right)$ and $g(p) \neq g(q)$. We consider the following three cases:

(1) $x_{p} \neq x_{q}$.

(2) $x_{p}=x_{q}, \tilde{f}(p)=\tilde{f}(q)$ whenever $\tilde{f} \in l^{\infty}(N, \operatorname{Re} A)$ vanishes on $N \times\left\{x_{p}\right\}$.

(3) $x_{p}=x_{q}$, there exists an $\tilde{f} \in l^{\infty}(N, \operatorname{Re} A)$ such that $\tilde{f}$ vanishes on $N \times\left\{x_{p}\right\}$ and $\tilde{f}(p) \neq \tilde{f}(q)$.

Case (1). Since $\operatorname{Re} A$ is uniformly dense in $C_{R}(X)$, there exists an $f \in \operatorname{Re} A$ such that $-1 \leqslant f\left(x_{p}\right) \leqslant 0, f\left(F_{1}\right) \subset[-1,0]$ and $f\left(x_{q}\right) \notin h^{-1}(0)$. Then $g=\overline{h \circ f}(\overline{h \circ f}=$ $\left(u_{n}\right)$ where $u_{n}=h \circ f$ for all $n$ ) is the desired function.

Case (2). $\operatorname{Re} A$ is dense in $C_{R}(X)$ and $h=0$ on $[-1,0]$, so there exists a $u \in \operatorname{Re} A$ such that $u\left(F_{0}\right)=1$ and $u\left(F_{1}\right)=0$. Since $\operatorname{Re} A$ is ultraseparating, there exists a $G \in l^{\infty}(N, \operatorname{Re} A)$ which separates $p$ and $q$. For each $n$ let $c_{n}$ denote $G\left(n, x_{p}\right)$. Then $g=\left(c_{n} u\right)$ is the desired function.

Case (3). Without loss of generality we may assume that $\tilde{f}(p)>0$. Put $\alpha=$ $\sup \left\{\tilde{f}(\tilde{x}): \tilde{x} \in \beta\left(N \times F_{1}\right)\right\}$. Let $\tilde{w}=\tilde{f}-(\alpha+1) \bar{u}$, where $u \in \operatorname{Re} A$ is 0 on $F_{0}$ and 1 on $F_{1}$. Then $\tilde{w}\left(\beta\left(N \times F_{1}\right)\right)<0, \tilde{w}\left(N \times\left\{x_{p}\right\}\right)=0, \tilde{w}(p)>0$ and $\tilde{w}(p) \neq \tilde{w}(q)$. Let $D=\left\{u \in \operatorname{Re} A: u\left(x_{p}\right)=0, u\left(F_{1}\right) \subset[-1,0],-1<u<1\right\}$. For each $n$ let $D_{n}=\{u \in D: N(h \circ u)<n\}$. Then $D$ is closed in $\operatorname{Re} A$ and $D=\cup_{n=1}^{\infty} D_{n}$. So by the Baire category theorem, the closure of some $D_{n}$ has nonempty interior in $D$. 
Thus there are a $u_{0} \in D$, a positive integer $r$, and an $\varepsilon>0$ such that $U \cap D_{r}$ is dense in $U \cap D$ where $U=\left\{u \in \operatorname{Re} A: N\left(u-u_{0}\right)<\varepsilon\right\}$. We may assume that $-1<u_{0}<1$ and $u_{0}\left(F_{1}\right) \subset(-1,0)$. Let $W_{\varepsilon}=\left\{\tilde{u}=\left(u_{n}\right) \in l^{\infty}(N, \operatorname{Re} A): u_{n} \in D\right.$, $\left.\sup _{n} N\left(u_{n}-u\right)<\varepsilon\right\}$. Then $h \circ \tilde{u} \in \mathrm{Cl}\left(l^{\infty}(N, \operatorname{Re} A)\right.$ ) (the uniform closure of $l^{\infty}(N, \operatorname{Re} A)$ in $\left.C_{R}(\beta(N \times X))\right)$ whenever $\tilde{u} \in W_{\varepsilon}$. For an appropriately small number $t>0$, we have $\bar{u}_{0}+t \tilde{w} \in W_{\varepsilon}, h \circ\left(\bar{u}_{0}+t \tilde{w}\right)(p)=h \circ(t \tilde{w})(p) \neq$ $h \circ(t \tilde{w})(q)=h \circ\left(\bar{u}_{0}+t \tilde{w}\right)(q)$ and $-1<\bar{u}_{0}+t \tilde{w}<0$ on $\beta\left(N \times F_{1}\right)$. There exists a sequence $\left\{v^{k}\right\}$ in $l^{\infty}(N, \operatorname{Re} A)$, where $v^{k}=\left(v_{n}^{k}\right)\left(v_{n}^{k} \in D_{r} \cap U\right)$, such that $v^{k} \rightarrow \bar{u}_{0}+t \tilde{w}$ uniformly on $\beta(N \times X)$ as $k \rightarrow \infty$. For sufficiently large $k$, we obtain that $h \circ v^{k}(p) \neq h \circ v^{k}(q)$ and that $h \circ v^{k}$ vanishes on $\beta\left(N \times F_{1}\right)$. This function $h \circ v^{k}$ is the desired function.

3. Proof of the theorem. Suppose that $h$ operates weak-boundedly at $x$ i.e., there exist an $\varepsilon>0$, a $\delta>0$, a compact neighborhood $F_{x}$ of $x$ and a nonempty compact subset $F_{0}$ of $X$ such that $F_{0} \cap F_{x}=\varnothing$ and $h \circ u \in(\operatorname{Re} A)_{x}$ and $N_{x}(h \circ u)<\varepsilon$ whenever $u \in(\operatorname{Re} A)_{x}^{0}$ with $N_{x}^{0}(u)<\delta$. Suppose that it follows that $F_{x}$ is an interpolation set for $A$. Then each point of $X$, with finitely many exceptions, has a compact neighborhood which is an interpolation set for $A$ by Lemma 1 . Then we get $A=C(X)$. So it is sufficient to prove that $F_{x}$ is an interpolation set for $A$.

Let $V$ denote $\operatorname{Cl}\left(l^{\infty}\left(N,(\operatorname{Re} A)_{x}\right)\right)$. We construct an algebra contained in $V$ which contains the constants and separates the points of $\beta\left(N \times F_{x}\right)$. Then the StoneWeierstrass theorem will imply that $V=C_{R}\left(\beta\left(N \times F_{x}\right)\right)$, and by Bernard's lemma [1] $(\operatorname{Re} A)_{x}=C_{R}\left(F_{x}\right)$ so $A \mid F_{x}=C\left(F_{x}\right)$ by the theorem of Sidney and Stout [G].

Let $\tilde{u}_{1}=\left(u_{n}^{1}\right), \tilde{u}_{2}=\left(u_{n}^{2}\right), \tilde{u}_{3}=\left(u_{n}^{3}\right), \ldots, \tilde{u}_{m}=\left(u_{n}^{m}\right)$ be in $l^{\infty}\left(N,(\operatorname{Re} A)_{x}^{0}\right)$. For sufficiently small $\Delta$, let $\lambda_{\Delta}$ be a nonnegative $n$-times continuously differentiable function supported in $(-\Delta, \Delta)$ and with integral 1 . Let $\phi_{\Delta}$ denote the convolution

$$
\phi_{\Delta}(x)=\int_{-\Delta}^{\Delta} h(x-t) \lambda_{\Delta}(t) d t, \quad-1+\Delta<x<1-\Delta .
$$

$\phi_{\Delta}$ is $n$-times continuously differentiable and converges uniformly to $h$ on any compact subinterval of $(-1,1)$ as $\Delta$ tends to 0 .

There exist an $s_{0} \in(-\delta / 2, \delta / 2)$ and a $\Delta<\delta / 2$ such that $\phi_{\Delta}^{(m)}\left(s_{0}\right) \neq 0$. For if $\phi_{\gamma}^{(m)}=0$ on $(-\delta / 2, \delta / 2)$ for each small $\gamma$, then $\phi_{\gamma}$ is a polynomial of degree at most $m-1$ so $h$ is also a polynomial of degree at most $m-1$ on $(-\delta / 2, \delta / 2)$, which is a contradiction. For sufficiently small $s_{1}, s_{2}, s_{3}, \ldots, s_{m}$ we have

$$
h \circ\left(s_{0}+s_{1} u_{n}^{1}+s_{2} u_{n}^{2}+s_{3} u_{n}^{3}+\cdots+s_{m} u_{n}^{m}-t\right) \in(\operatorname{Re} A)_{x}
$$

and

$$
N_{x}\left(h \circ\left(s_{0}+s_{1} u_{n}^{1}+s_{2} u_{n}^{2}+s_{3} u_{n}^{3}+\cdots+s_{m} u_{n}^{m}-t\right)\right)<\varepsilon
$$

for each $n$ whenever $|t|<\Delta$. Thus

$$
h \circ\left(s_{0}+s_{1} \tilde{u}_{1}+s_{2} \tilde{u}_{2}+s_{3} \tilde{u}_{3}+\cdots+s_{m} \tilde{u}_{m}-t\right) \in V
$$

if $|t|<\Delta$, so

$$
\phi_{\Delta} \circ\left(s_{0}+s_{1} \tilde{u}_{1}+s_{2} \tilde{u}_{2}+s_{3} \tilde{u}_{3}+\cdots+s_{m} \tilde{u}_{m}\right) \in V .
$$


In particular fixing $s_{2}, s_{3}, \ldots, s_{m}$ and varying $s_{1}$ gives

$$
\phi_{\Delta} \circ\left(s_{0}+s_{2} \tilde{u}_{2}+s_{3} \tilde{u}_{3}+\cdots+s_{m} \tilde{u}_{m}\right) \in V
$$

hence

$$
\begin{aligned}
\left\{\phi _ { \Delta } \circ \left(s_{0}+s_{1} \tilde{u}_{1}+s_{2} \tilde{u}_{2}\right.\right. & \left.+\cdots+s_{m} \tilde{u}_{m}\right) \\
& \left.-\phi_{\Delta} \circ\left(s_{0}+s_{2} \tilde{u}_{2}+\cdots+s_{m} \tilde{u}_{m}\right)\right\} / s_{1} \in V
\end{aligned}
$$

if $s_{1}$ is small and nonzero, and letting $s_{1} \rightarrow 0$,

$$
\phi_{\Delta}^{\prime} \circ\left(s_{0}+s_{2} \tilde{u}_{2}+\cdots+s_{m} \tilde{u}_{m}\right) \tilde{u}_{1} \in V
$$

for small enough $s_{2}, s_{3}, \ldots, s_{m}$. Continuing in this manner, in $m$ stages we get

$$
\phi_{\Delta}^{(m)}\left(s_{0}\right) \tilde{u}_{1} \cdot \tilde{u}_{2} \cdot \tilde{u}_{3} \cdots \cdot \tilde{u}_{m} \in V .
$$

Therefore the algebra generated by $l^{\infty}\left(N,(\operatorname{Re} A)_{x}^{0}\right)$ separates the points of $\beta\left(N \times F_{x}\right)$, hence this algebra is the desired algebra.

The author wishes to thank Professor Junzo Wada for helpful discussions and for his constant encouragement.

\section{REFERENCES}

1. A. Bernard, Espaces des parties réelles des éléments d'une algèbre de Banach de fonctions, J. Funct. Anal. 10 (1972), 387-409.

2. A. Bernard and A. Dufresnoy, Calcul symbolique sur la frontière de Silov de certaines algèbres de fonctions holomorphes, Ann. Inst. Fourier (Grenoble) 25 (1975), 33-43.

3. R. Burckel, Characterizations of $C(X)$ among its subalgebras, Dekker, New York, 1972.

4. K. de Leeuw and Y. Katznelson, Functions that operate on non-selfadjoint algebras, J. Analyse Math. 11 (1963), 207-219.

5. S. Sidney, Functions which operate on the real part of a uniform algebra, Pacific J. Math. 80 (1979), 265-272.

6. S. Sidney and L. Stout, A note on interpolation, Proc. Amer. Math. Soc. 19 (1968), 380-382.

7. J. Wermer, The space of real parts of a function algebra, Pacific J. Math. 13 (1963).

Department of Mathematics, Waseda UNIVERSITY, Tokyo, JaPAN 